\title{
A Study of Energy Recovery System during Braking for Electric
}

\author{
Vehicle \\ Zheng Lin ${ }^{1, a^{*}}$, Shi Zhanqun ${ }^{2, b}$, Luo Yuegang ${ }^{3, c}$ and Kang Jing ${ }^{4, d}$ \\ ${ }^{1}$ Dalian Minzu University, No. 18, Liaohe West Road, Jinzhou New District, Dalian, Liaoning \\ Province, 116600, P.R. China \\ ${ }^{2}$ Hebei University of Technology, Xiping Road No. 5340, Beichen District, Tianjin, 300130, \\ P.R.China

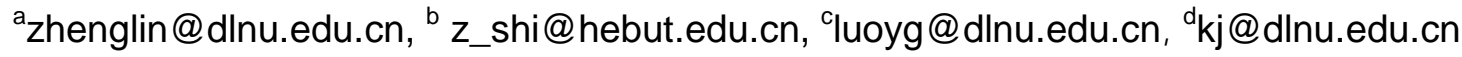

Keywords: Energy Regenerative System, Electric Vehicle, Braking System

\begin{abstract}
The braking energy recovery system of electric vehicle is worthy to be further discussed due to the improvement of both the energy efficiency and the endurance capacity. In this paper, the structural and the principle of the energy regenerative system for electric vehicles are discussed. The comparisons between the conventional braking system and the regenerative braking system are necessary in order to improve the advantages by using the energy regenerative system.
\end{abstract}

\section{Introduction}

Under the consider of energy crisis and environmental pollution problems have become more severe, new energy vehicles has become a hot research field. A battery electric vehicle (BEV), battery-only electric vehicle (BOEV) or all-electric vehicle is a type of electric vehicle (EV) that uses chemical energy stored in rechargeable battery packs[1].

The anti-lock braking system (ABS) is well known and commonly employed in conventional internal combustion engine vehicles [2]. ABS aims to brake a vehicle travelling at a high speed in such a way that the control of the vehicle always remains in the hands of driver and the wheels do not get locked up [3].

The energy recovery braking is also called regenerative braking. If the kinetic energy of electric vehicle can be transformed into electrical energy by the regenerative braking system and recovered into the storage system during vehicle braking process, the energy efficiency of the electric vehicle will be improved, and the driving range of the electric vehicle will be extended [4].

\section{Structural and principle of the regenerative braking system}

The braking system of electric vehicle is part the same as traditional vehicle. The purpose of braking system is to reduce the speed or to stop the vehicle. The traditional braking system includes the braking components and the control system. Generally speaking, besides the traditional braking system, the regenerative braking system is also loaded on the electric vehicle. By using the control circuits of driving motor to regenerate energy for the motor, the deceleration kinetic energy is going back to the batter to complete a whole regenerative braking circle [5].

Fig. 1 shows the structural and the principle of the regenerative braking system. The regenerative braking control unit is acting the role as the central controller in the general control system. The regenerative braking control unit is formally independent, but work in mutual coordination states with the motor control unit. 

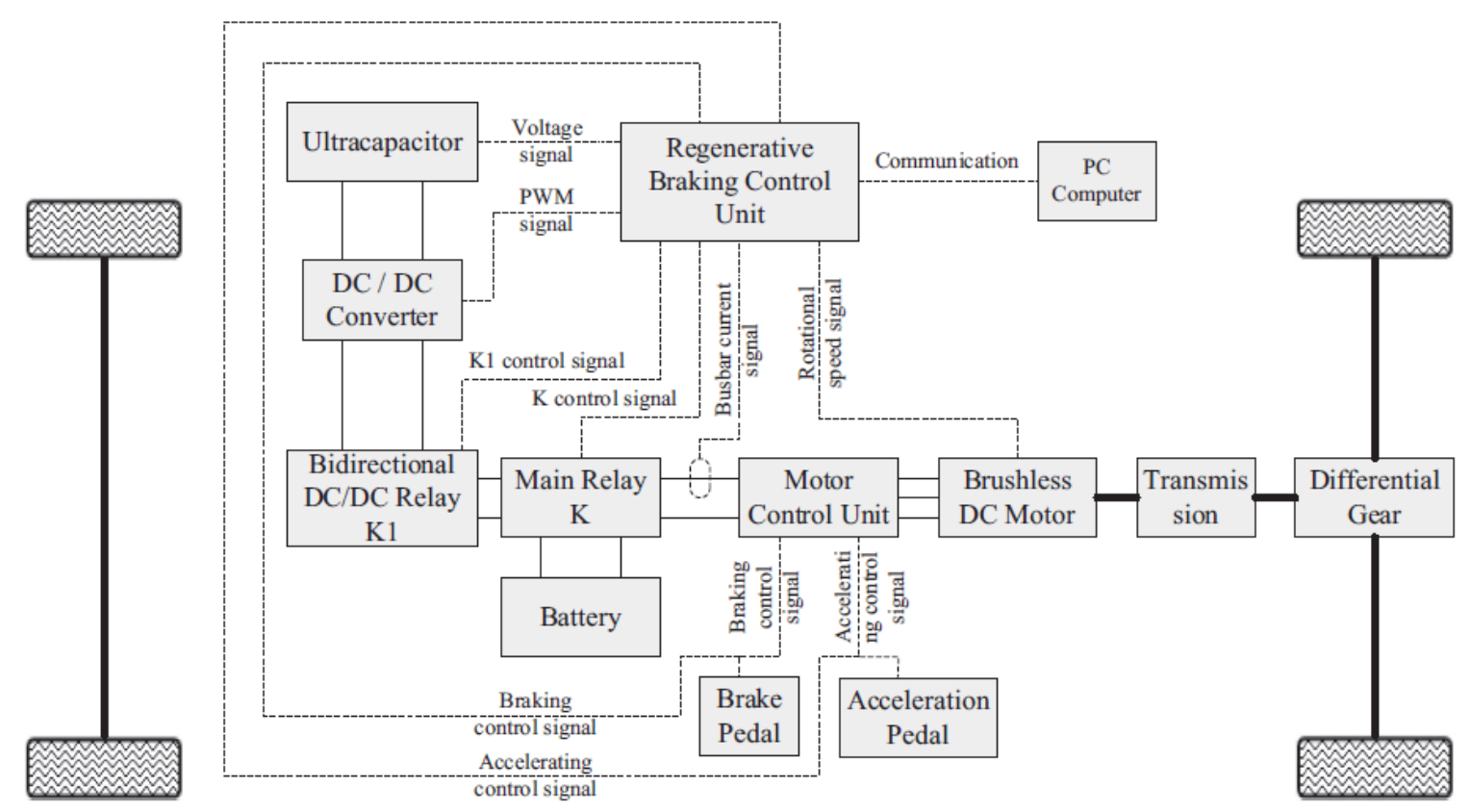

Fig. 1 Structure diagram of regenerative braking control system [4]

The control strategies of electric vehicle regenerative braking system include the following rules [6,7]:

(1) Get ideal braking force

(2) Best braking energy recovery

(3) Parallel braking energy recovery

(4) Minimize the energy use during braking

(5) Conserve the available energy stored in battery

The functions that implemented by regenerative braking control system include the following aspects $[4,8]$ :

(1) Switching control of the main circuit

(2) Identification of the driver intention

(3) Regenerative braking control

(4) Through the communication between the control system and the computer, the operating state signals of the regenerative braking control system are displayed and stored by the computer in real-time.

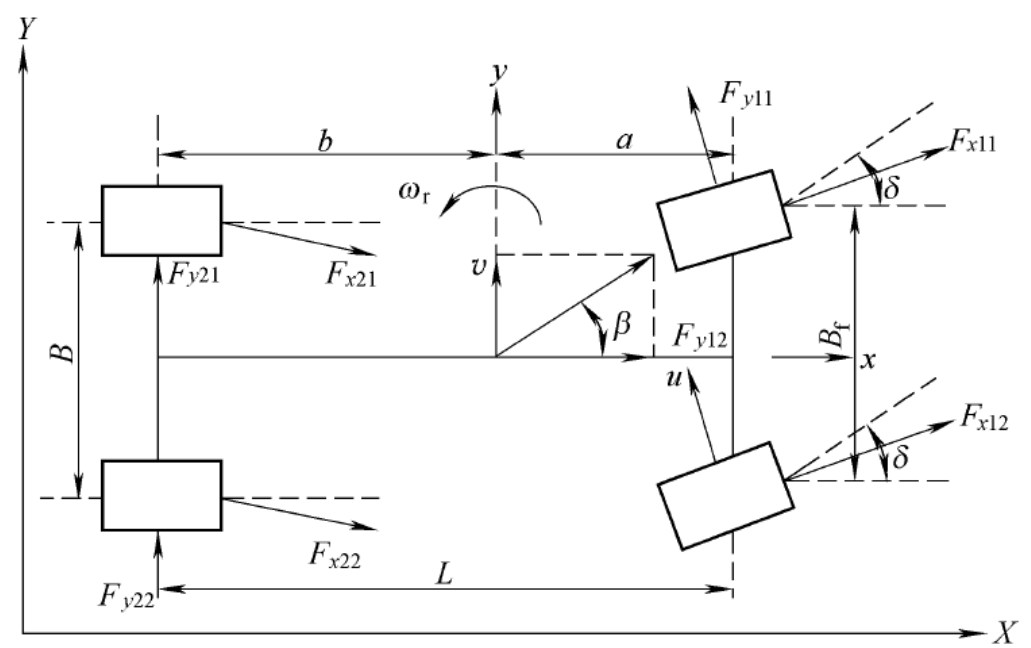

Fig.2 Vehicle dynamics model [2]

Fig. 2 shows the dynamic model of vehicle. Table 1 shows the instructions of symbols used in Fig.2. Fig. 3 describes the control block diagram of signals transmission. 
Table 1 Instruction of symbols of Fig.2

\begin{tabular}{|c|c|}
\hline Symbol & Instruction \\
\hline $\mathrm{F}_{\mathrm{x}}$ & Longitudinal force of tyre \\
\hline $\mathrm{F}_{\mathrm{y}}$ & Lateral force of tyre \\
\hline $\mathrm{F}_{\mathrm{f}}$ & Rolling resistance \\
\hline $\mathrm{B}_{\mathrm{f}}$ & Distance between two front tyres \\
\hline $\mathrm{B}$ & Distance between two rear tyres \\
\hline$\delta$ & Front steer angle \\
\hline $\mathrm{a}$ & Distance between the center of mass and the front axis \\
\hline $\mathrm{b}$ & Distance between the center of mass and the rear axis \\
\hline $\mathrm{L}$ & Distance between the front and rear tyres \\
\hline$\omega_{\mathrm{r}}$ & Wheel angular velocity \\
\hline $\mathrm{V}$ & Wheel linear velocity \\
\hline
\end{tabular}

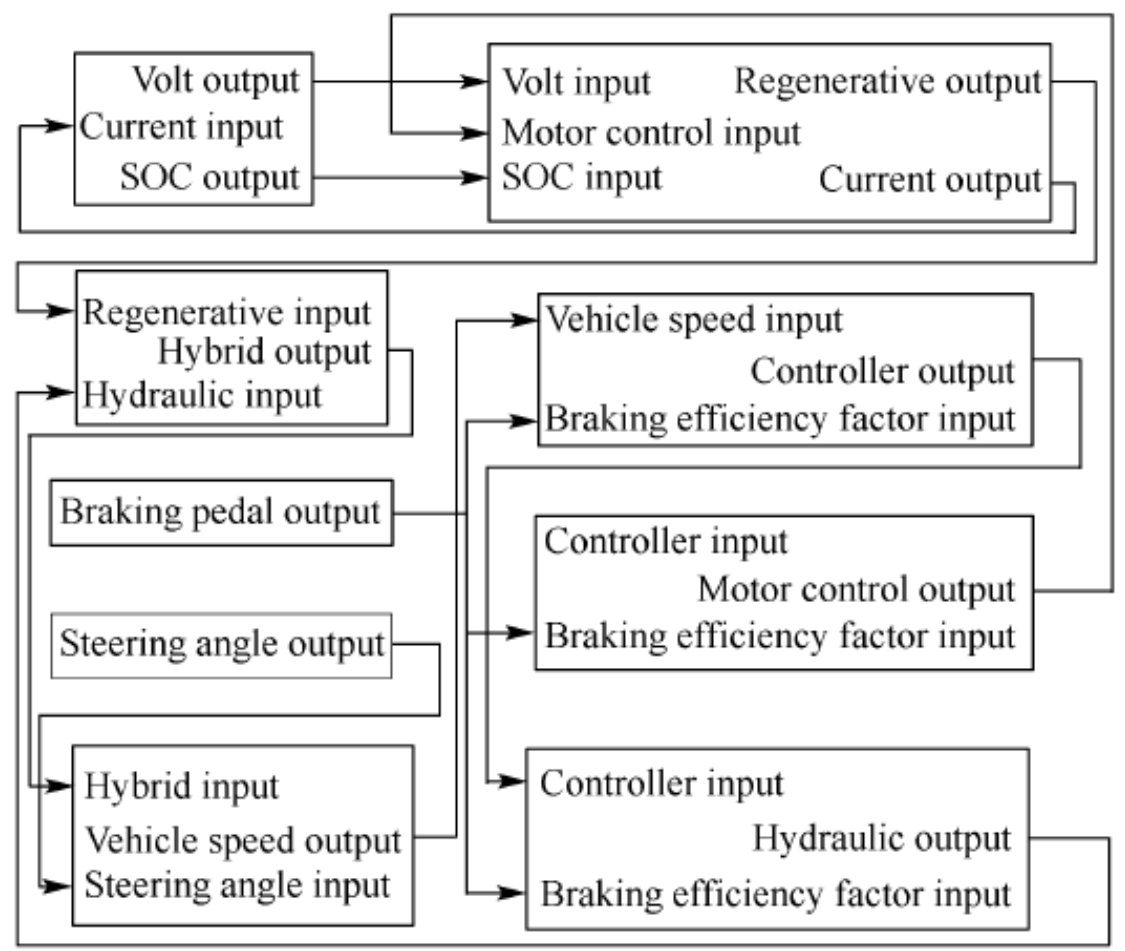

Fig.3 Diagram of the control signals transmission [2]

\section{Distribution relationship between the friction braking force and the motor braking force}

The braking force is composed of two parts in the electric vehicle braking system. Part of the braking force is the friction force that provided by conventional air or hydraulic braking system. The other part of the braking force is the energy recovery braking force that provided by the motor. Figure 4 shows the relationship of the braking force distribution. Under the premise of getting the 
maximum energy recovery, coordinating the distribution relationship between the motor and the mechanical braking system, is the core of braking control strategy of electric vehicle.

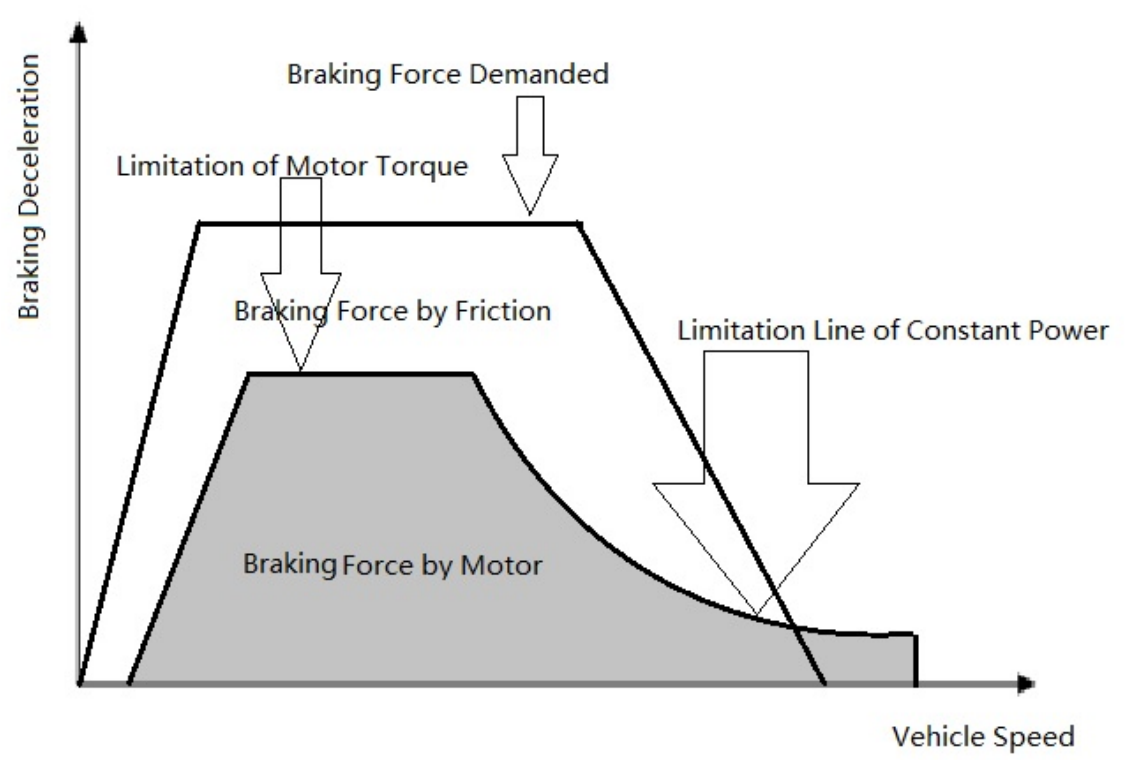

Fig.4 Schematic Diagram of Braking Force Distribution

The general braking force $F_{\text {general }}$ is generated by the hydraulic friction braking force $F_{f}$ and the motor braking force $\mathrm{F}_{\mathrm{m}}$.

$\mathrm{F}_{\text {general }}=\mathrm{F}_{\mathrm{f}}+\mathrm{F}_{\mathrm{m}}$

The declaration of speed is

$\frac{\mathrm{du}}{\mathrm{dt}}=\frac{\mathrm{F}_{\mathrm{f}}+\mathrm{F}_{\mathrm{m}}+\mathrm{F}_{1}}{\mathrm{~m}}$

where $\mathrm{F}_{1}$ is the braking for lost during braking and $\mathrm{m}$ is the quality of vehicle.

Generally speaking, the braking process of vehicle can be classified into three types as follows:

(1) Emergency braking

The deceleration of speed is over $4 \mathrm{~m} / \mathrm{s}^{2}$ in the emergency braking case. There is no necessary to recover energy during emergency braking due to the braking time limitation. The braking force is mainly based on the hydraulic friction braking force $F_{f}$, rather than the motor braking force $\mathrm{F}_{\mathrm{m}}$.

(2) Normal braking

The main braking force is provided by the motor braking force $\mathrm{F}_{\mathrm{m}}$. Only when the motor could not provide enough braking force, the hydraulic friction braking force $F_{f}$ is going to work. Therefore, the energy recovery system can recovered more energy under normal braking process.

(3) Long downhill retarding braking

The demand of braking force is not too much during long downhill retarding braking process. The motor braking force $F_{m}$ could provide enough braking force in this case.

\section{Comparisons between regenerative braking and normal braking}

Presupposing the start of a brake pedal during braking, the normal braking mode starts firstly. According to the state of the vehicle and the information of wheels, the braking mode switching starts. Based on the slip ratios of wheels, the braking mode switches between normal braking, ABS braking and regenerative braking. The entire braking mode switching process is as shown in Fig. 5. 


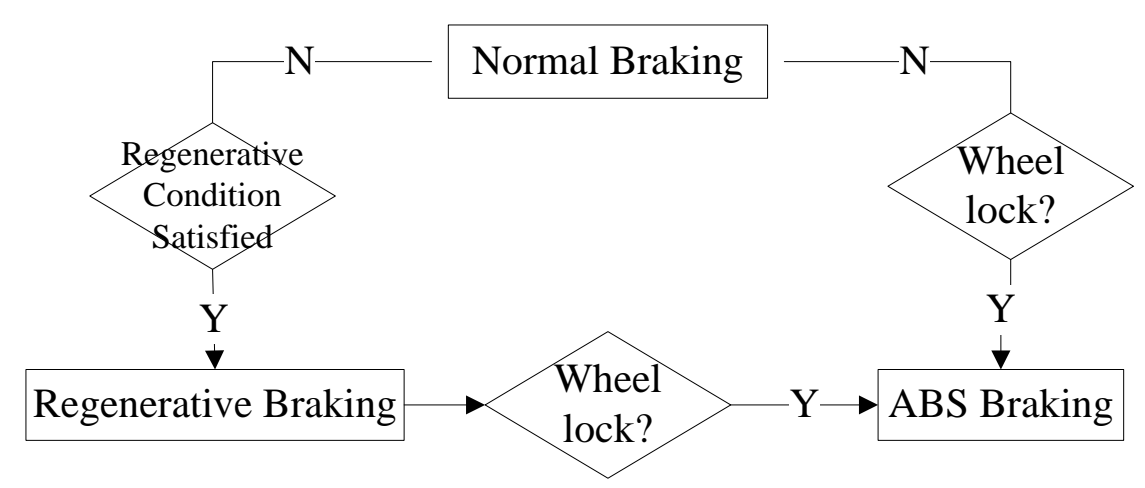

Fig. 5 Braking mode switching process

The comparisons between regenerative braking and normal braking are discussed as follows.

(1) The energy efficiency

In normal braking situation, the friction braking force is converted into heat finally. In regenerative braking situation, part of the mechanical energy is converted into electrical energy, which stored in the energy storage elements for reuse. In this case, the energy efficiency is increased.

(2) Braking reliability

When the electric vehicle in long downhill retarding braking situation, the temperature of brakes' surface rises due to the frequently brakes. The instability rises at the same time. On the other hand, when the vehicle in regenerative braking situation, the frequency of friction brake is reduced efficiently, which reduces the temperature of brakes' surface in the meantime. By using the regenerative braking, both the braking performance and the braking safety are improved effectively.

(3) Endurance capacity

In normal braking situation, the braking process is converted into kinetic energy through friction. Such part of energy is lost as heat. In regenerative braking situation, this part of energy can be absorbed and then to be reused. In this case, the endurance capacity is improved significantly.

(4) Maintenance costs

In normal braking system, the brake pads required to be replaced frequently due to the usage of friction braking mode. In this case, the maintenance costs of vehicle are increased somehow. However, in regenerative braking system with brake energy recovery components, the frequency of using the friction brakes is reduced effectively. In this case, the maintenance costs are reduced obviously.

\section{Summary}

In this work, the structural and principle of regenerative braking system of electric vehicle are discussed firstly. Based on the comparison results between the conventional braking system and the regenerative braking system of electric vehicle, the distribution relationship are listed in diagrams. Both the advantages and disadvantages of the regenerative braking system are analyzed. This work figures out the direction for the development of braking energy regenerative braking system of electric vehicle in the future.

\section{Acknowledgements}

This work is supported by the "Fundamental Research Funds for the Central Universities" (No. DC20150201402, No. DC201502010202), the "2013 Liaoning Province Major Construction of Undergraduate Engineering Talents Training Mode", the "Foundation of Liaoning Education Department” (No. L20145454) and the “Key Project of Tianjin NSFC”(No. 13JCZDJC3400). 


\section{References}

[1] Wikipedia, Battery electric vehicle, https://en.wikipedia.org/wiki/Battery_electric_vehicle

[2] Zhang Jianlong, Yin Chengliang, and Zhang Jianwu, Design and Analysis of Electro-mechanical Hybrid Anti-lock Braking System for Hybrid Electric Vehicle Utilizing Motor Regenerative Braking [J], Chinese Journal of Mechanical Engineering, Vol.22, No.1, 2009

[3] S Maliye, P Satapathy, S Kumar, K Mahapatra, Regenerative and Anti-Lock braking system in electric vehicles [C], 2014 IEEE International Conference on Advanced Communication Control and Computing Technologies (ICACCCT), pp.1019-1023.

[4] Chaofeng Pan, Liao Chen, Long Chen, Haobin Jiang, Zhongxing Li, Shaohua Wang, Research on motor rotational speed measurement in regenerative braking system of electric vehicle, Mechanical Systems and Signal Processing [J], 66-67 (2016), pp. 829-839

[5] Liu Chongyu, Research of Control Strategy and Simulation of Pure Electric Vehicle's Regenerative Braking [D], Master’s Dissertation, Wuhan University of Technology, 2010

[6] Gao Yimin, Chen Liping, Mehrdad Ehsani, Investigation of the Effectiveness of Regenerative Braking for EV and HEV [J], SAE, 1990-01-2910

[7] A.M.Walker, M.U.Lamperth, S.Wilkins, On Friction Braking Demand with Regenerative Braking [J], SAE 2002-01-2581

[8] Tian Feng, liu Haiyang, Tang Panpan, Braking Energy Recovery System for Electric Vehicle, Automobile Applied Technology, 2015 No.2, pp.47-50 